\title{
Read alignment
}

All reads were aligned to the human reference genome hg19 (GRCh37.66) using the Burrows-Wheeler Aligner[2]. In the case of data originating from the 454 and the Illumina NextSeq sequencer, BWA mem (version 0.7.8) was used. In the case of the Ion Torrent data, TMAP (version 3.0.1, [1], http://github.com/iontorrent/tmap) was used, which is an Ion Torrent specific version of BWA.

\section{References}

[1] Homer N, Lyons M, Shah M. (2010) TMAP: the torrent mapping program.

[2] Li H, Durbin R. (2009) Fast and accurate short read alignment with Burrows-Wheeler transform, Bioinformatics, 25, 1754-1760. 